\title{
Preoperative serum fibrinogen as a valuable predictor in the nomogram predicting overall survival of postoperative patients with gallbladder cancer
}

\author{
Ziyi Yang ${ }^{1,2 \#}$, Tai Ren ${ }^{2,3 \#}$, Shilei Liu ${ }^{1,2}$, Chen Cai $^{1,2}$, Wei Gong ${ }^{1,2}$, Yijun Shu ${ }^{1,2}$ \\ ${ }^{1}$ Department of General Surgery, Xinhua Hospital Affiliated to Shanghai Jiao Tong University School of Medicine, Shanghai, China; ${ }^{2}$ Shanghai Key \\ Laboratory of Biliary Tract Disease Research, Shanghai, China; ${ }^{3}$ Ministry of Education - Shanghai Key Laboratory of Children's Environmental \\ Health, Xinhua Hospital Affiliated to Shanghai Jiao Tong University School of Medicine, Shanghai, China \\ Contributions: (I) Conception and design: Z Yang, T Ren, W Gong, Y Shu; (II) Administrative support: W Gong; (III) Provision of study materials or \\ patients: W Gong, Y Shu; (IV) Collection and assembly of data: Z Yang, T Ren, S Liu, C Cai; (V) Data analysis and interpretation: Z Yang, T Ren; (VI) \\ Manuscript writing: All authors; (VII) Final approval of manuscript: All authors. \\ \#These authors contributed equally to this work. \\ Correspondence to: Wei Gong; Yijun Shu. Department of General Surgery, Xinhua Hospital Affiliated to Shanghai Jiao Tong University School of \\ Medicine, No. 1665 Kongjiang Road, Shanghai 200092, China. Email: gongwei@xinhuamed.com.cn; shuyijun19881125@163.com.
}

Background: Coagulation and fibrinolysis activation are frequently observed in cancer patients, and the tumors in these cases are thought to be associated with a higher risk of invasion, metastasis and worse longterm outcome. The specific aim of this study was to develop an effective prognostic nomogram to help make individualized estimates for patients with resected gallbladder cancer (GBC).

Methods: Patients with resected GBC who were diagnosed between 2006 and 2014 at Xinhua Hospital were selected. Model performance was measured by c-index and calibration curve. The results were further validated using bootstrap and a cohort of 38 patients from a branch hospital who underwent surgery from 2006 to 2014.

Results: Backward stepwise selection and Lasso were applied respectively to select predictors. T stage, $\mathrm{N}$ stage, and preoperative serum fibrinogen were included in the final model. Predictions correlated well with observed 1 - and 3-year survival. The c-index for predicting survival was 0.74 (95\% confidence interval, 0.70-0.78), which was statistically higher than that of the AJCC 7th system and Nevin system ( $\mathrm{P}=0.04,0.04$, respectively). In the validation cohort, the nomogram performed better than the other two staging systems (c-index: 0.71 vs. 0.67 and 0.67 ).

Conclusions: The validated nomogram is a practical tool for predicting the overall survival (OS) of postoperative GBC patients. Preoperative serum fibrinogen levels were associated with tumor progression and may be an independent predictor for GBC patients.

Keywords: Clinical prediction model; fibrinogen; gallbladder cancer (GBC); nomogram

Submitted May 07, 2021. Accepted for publication Aug 17, 2021.

doi: 10.21037/jgo-21-357

View this article at: https://dx.doi.org/10.21037/jgo-21-357

^ ORCID: Ziyi Yang, 0000-0001-5883-9552; Tai Ren, 0000-0001-5864-9425. 


\section{Introduction}

Gallbladder cancer (GBC) is the most common biliary tract malignancy, and also the sixth most common gastrointestinal malignancy, with an annual incidence of 2.5-4.0 per 100,000 $(1,2)$. The median survival time for GBC patients is less than 6 months, with a 5 -year survival rate of $5 \%(3,4)$. Several factors contribute to its dismal prognosis, including late detection, rapid metastasis, and early acquired resistance to chemotherapy $(5,6)$. Surgery is the only curative therapy, though many of the patients lose the opportunity because of late detection (7).

Cancer-induced coagulation abnormalities have been recognized as linked to several malignancies $(8,9)$. Fibrinogen, an essential part of the coagulation system, has been reported to be related with human malignancies such as renal cell carcinoma, head and neck cancer, colorectal (10), endometrial (11), pancreatic (12), and GBC (13). Though the underlying mechanism has not been fully elucidated, the potential ability to predict the postoperative prognosis of GBC patients should be taken into consideration.

Due to the low incidence of GBC, no large-scale clinical trials of predicting the prognosis of GBC patients have been carried out, resulting in little evidence to rely on when predicting the prognosis of patients after surgery. The 7th AJCC staging system has shown good discrimination, but modest accuracy (14). Independent prognostic factors other than $\mathrm{T}$ stage, $\mathrm{N}$ stage, and $\mathrm{M}$ stage have been identified, including concomitant gallstones, anomalous pancreaticobiliary ductal junction, histologic differentiation, preoperative total bilirubin, CEA, CA199, CA125, and fibrinogen (3,13,15-18). However, these potential predictors have not been evaluated systematically, and thus are not applicable for clinical use. Combining these factors in a prediction model is likely to enhance the accuracy of survival predictions for patients after resection of GBC. More accurate predictions for patients may help to identify high-risk groups, potentially leading to a stratified treatment strategy.

The nomogram is a practical tool that integrates biological and clinical features for clinical prediction, with a user-friendly interface and increased accuracy (19). In recent years, nomograms have been applied to clinical prediction models for various types of malignancies (20-23). This study aimed to build a predictive nomogram for GBC patients undergoing resection, based on two independent institutional cohorts. We present the following article in accordance with the TRIPOD reporting checklist (available at https://dx.doi.org/10.21037/jgo-21-357).

\section{Methods}

\section{Study design and participants}

As shown in Figure 1, the prediction model was developed in multiple steps. The nomogram was developed based on a retrospective cohort $(\mathrm{n}=174)$ of patients who underwent surgical resection for GBC between January 2006 and September 2014 at Xinhua Hospital, Shanghai, China; a patient cohort $(n=38)$ from the branch hospital in Chongming was used for external validation. Inclusion criteria included: no history of previous anticancer therapy; no history of other malignancies; complete resection of macroscopic tumor; and histologically confirmed GBC. Exclusion criteria were: perioperative death, and emergency surgery.

The study was censored on September 30, 2017. All procedures performed in this study involving human participants were in accordance with the Declaration of Helsinki (as revised in 2013). It was approved by the Committee for Ethics of Xinhua Hospital Affiliated to Shanghai Jiao Tong University School of Medicine (No. XHEC-C-2018-174). Informed consent was given before surgery.

\section{Diagnosis and treatment}

Blood samples were collected after routine history taking and physical examination. Liver function and coagulation tests were performed 2-3 days before surgery. Malignancy was either diagnosed incidentally on pathologic examination after a simple cholecystectomy, or discovered with preoperative computed tomography or magnetic resonance imaging/magnetic resonance cholangiopancreatography. Surgery was indicated when the tumor was discovered, except if preoperative tests indicated distant metastasis, inoperable primary tumor, or multiple malignancies. Malignancy was then confirmed by histological examination.

Simple cholecystectomy was performed for patients with T1 lesions; for T2-T4 lesions, radical resection was routinely performed, including en-bloc gallbladder resection, resection of a 2-cm liver wedge, and combined resection of infiltrated organs. Lymph node dissection was performed in selected cases. The "curettage and aspiration dissection technique" was applied with Peng's multifunctional operative dissector (24). 


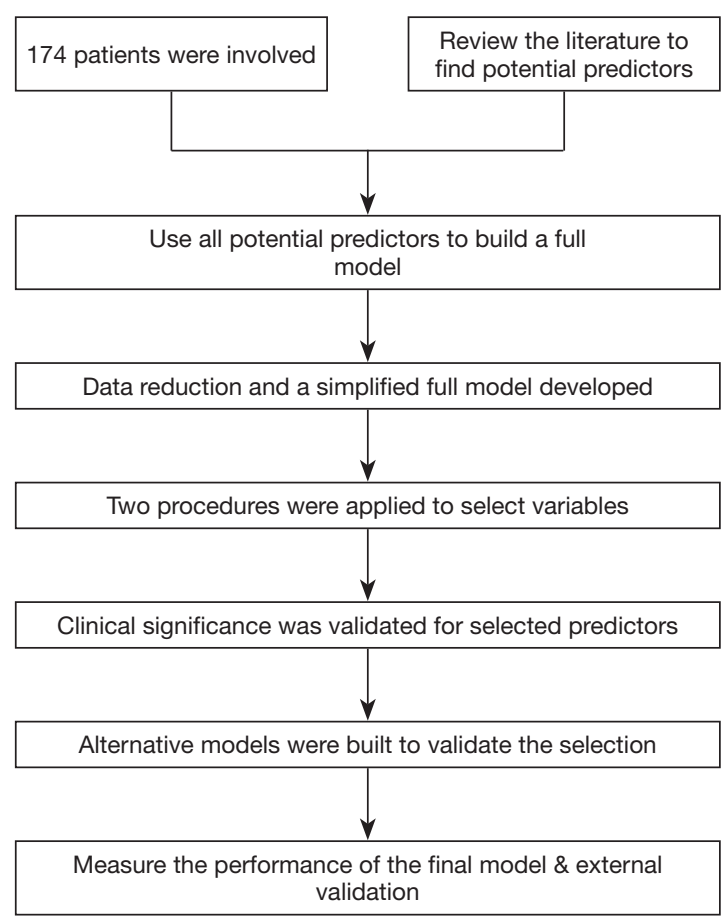

Figure 1 Flowchart of developing the prediction model.

Tumors histologically diagnosed after cholecystectomy were termed incidental GBC. For these patients, if T stage $>1$, a second radical operation was recommended.

\section{Follow-up}

Patients were followed postoperatively every 3 months for the first 2 years, and then every 6 months thereafter. Overall survival (OS) were used as the primary endpoint, defined as the interval between the date of surgery and death or the last date of follow-up (September 30, 2017). When a secondary surgery was performed, the start point was defined as the date of the first operation.

\section{Variables}

After a thorough review of the literature, potential predictors were selected with reference to their statistical significance for prognosis, data accessibility, and cost effectiveness. Potential predictors were selected as follows: basic patient demographics (age and sex), R0 resection or not, histologic differentiation, TNM stage, concomitant cholelithiasis, tumor markers (CA-199, CA-125, and CEA), liver function (ALT), indicator of jaundice (TBIL), and coagulation indicators (fibrinogen and INR). The staging and histologic grading was based on the 7 th edition of the AJCC manual (25). The operation year was included in the model development procedure to exclude the possible effect of improvement in surgical techniques and equipment during the time span.

\section{Statistical analysis}

Statistical analyses were performed using R software, version 3.4.3. Before modeling, the categorical variables were grouped based on clinical findings. Continuous variables were compared using the $t$-test or Mann-Whitney $\mathrm{U}$ test for variables with an abnormal distribution. Discrete variables were compared using the $\chi^{2}$ test. $\mathrm{KM}$ and log-rank tests were applied to depict survival curves. Cox regression analysis was used for the model development.

Prediction models were formulated using the package of rms, version 5.1-2 (26,27). The model was developed according to checklist described previous research (28) (Table S1). Discrete variables were treated as binary, and continuous variables were fitted to restricted cubic spline function, $\log$ function, squared function, or linear function, depending on the pattern on data inspection. The pattern of missing data was inspected and analyzed, then the missing data were input with simple imputation. The full model was established with all potential predictors, then the predictors were selected with reference to a backward step-down selection procedure with the AIC and the Lasso model. Clinical significance was verified with each selected predictors by KM curve. The model performance was assessed using concordance probabilities (c-statistic). Finally, model calibration was checked in the derivation and external validation cohorts. Package survminer (version 0.4.2) was applied to draw the KM curves (29). Package glmpath (version 0.98) was applied to do the Lasso test (30). $\mathrm{P}<0.05$ was considered statistically significant. Package tidyverse (version 1.2.1) was applied to inspect, explore and analyze the data (31).

\section{Results}

\section{Clinicopathologic characteristics of the patients}

Our model was developed based on the primary cohort; a total of 174 patients met the inclusion criteria. In the validation cohort, 38 patients from another center (branch hospital in Chongming) were included with the same 
criteria. The detailed inspection and comparison of baseline characteristics between the two groups are shown in Table 1 . The general pattern of disease was comparable with the literature. The number of missing data was limited for all the predictors $(<1 \%)$, possibly because all the predictors selected were results from routine tests even 10 years ago. Missing data showed no systematic pattern (data not shown), and simple imputation was applied to the missing values.

The median follow-up time was 13 months (range, 2-83 months). The median OS was 12 months, and the 1and 3 -year OS rates were $64 \%$ and $17.2 \%$, respectively, better but still dismal results compared with data from the literature (3).

\section{Model development}

\section{Development of the full model}

The full model was developed with all potential predictors, including age, sex, R0 resection or not, level of histologic differentiation, T stage (7th AJCC), N stage (7th AJCC), concomitant cholelithiasis, tumor markers (CA-199, CA125, and CEA), ALT, total bilirubin, fibrinogen, and INR. Discrete variables were treated as dummy variables, and continuous variables were fitted to 3-knots restricted cubic spline functions (Table S2). According to the generally accepted rule (26), in order to balance the reliability and overfitting of a model, the degrees of freedom in the final model should be no more than the number of events/15, namely, $112 / 15 \approx 7$. Therefore, reduction in the degree of freedom was necessary. According to the effect of each predictor on the log hazard of death (Figure S1), together with the parameters derived from the full model, we manually merged the discrete variables with a similar effect on prognosis, then the continuous variables were either kept with the restricted cubic spline function or simplified to be linear. The simplified full model was then developed (Table S3).

As suggested (32), radical GBC surgery should involve at least six lymph nodes for adequate staging and stratifying recurrence risk. Here we meant to divide the group with this indicator. However, few of the patients met this criterion, so this variable was classified as either positive or 'not available'.

\section{Variable selection and development of the final model}

We applied a backward stepwise selection procedure based on the AIC and a Lasso procedure, respectively, to select the predictors from the simplified model. The two methods showed comparable results, indicating $\mathrm{T}$ stage, lymph node positivity, and preoperative fibrinogen as effective and valid predictors for prognosis. CA125 was selected in the Lasso model. However, the coefficient of CA125 was $<0.001$, indicating a negligible effect on the result. Taking the two results together, T stage, lymph node positivity, and preoperative fibrinogen were selected in the final model. The detailed selection results are shown in Table S3. The procedure of Lasso is shown in Figure 2.

After variable selection, the proportional hazard test was performed to examine the validity of the essential presumptions of Cox regression. The result confirmed the model as valid (Table S4). Redundancy analysis of the three selected predictors in the final model was performed. The result showed little redundancy among the three variables, indicating little inter-dependency of the variables (Table S5). Next, we assessed the interactions between each pair of selected variables by developing corresponding regression models (e.g., $\mathrm{T}+\mathrm{N}+\mathrm{Fib}+\mathrm{T} \times$ $\mathrm{N})$. No positive results were found (data not shown). Thus the final model was specified (Table 2).

\section{Serum fibrinogen as an independent prognostic factor}

In a prediction model, clinical meaningfulness is as important as statistical significance (26). The $\mathrm{T}$ and $\mathrm{N}$ stages are canonical indicators that have been long observed to be related with patient prognosis. Fibrinogen, however, was selected prior to other predictors, including the level of differentiation and tumor makers, which are generally thought to be more relevant to malignancy. Following this, we inspected and testified the significance of preoperative serum fibrinogen as an independent and valuable prognostic factor.

\section{Diagnostic performance of fibrinogen for prognosis}

With the fibrinogen level correlating with GBC prognosis, the diagnostic performance of fibrinogen for tumor staging and prognosis in patients with GBC was further investigated. We found that the AUC of the fibrinogen level was 0.671 (for convenience, only 3-year prognosis were used for the following prediction, Figure S2). Based on the ROC curve, a cut-off value of $4.43 \mathrm{~g} / \mathrm{L}$ was selected to dichotomize the cohort; thus, a plasma fibrinogen level $>4.43 \mathrm{~g} / \mathrm{L}$ were defined as hyperfibrinogenemia. The resultant PPV was 0.71 , indicating that hyperfibrinogenemia may be a reliable threshold for predicting the prognosis. 
Table 1 Patients' characteristics

\begin{tabular}{|c|c|c|}
\hline Demographic and clinicopathologic characteristics of included patients & Xinhua Hospital $(\mathrm{N}=174)$ & Chongming Branch Hospital $(\mathrm{N}=38)$ \\
\hline Age, years (median, range) & 67 [28-91] & $67[42-90]$ \\
\hline \multicolumn{3}{|l|}{ Differentiation } \\
\hline Well & $19(10.9 \%)$ & $5(13.2 \%)$ \\
\hline Poor & $59(33.9 \%)$ & $18(47.4 \%)$ \\
\hline \multicolumn{3}{|l|}{ T stage, 7th ed AJCC } \\
\hline Tis & $12(6.9 \%)$ & $3(7.9 \%)$ \\
\hline $\mathrm{T} 1$ & $12(6.9 \%)$ & $2(5.3 \%)$ \\
\hline $\mathrm{T} 4$ & $21(12.1 \%)$ & $2(5.3 \%)$ \\
\hline \multicolumn{3}{|l|}{ Lymph node } \\
\hline Positive & $63(36.2 \%)$ & $12(31.6 \%)$ \\
\hline Negative, $\geq 6$ nodes examined & $7(4.0 \%)$ & $5(13.2 \%)$ \\
\hline Negative, $<6$ nodes examined or data not available & $104(59.8 \%)$ & $21(55.3 \%)$ \\
\hline \multicolumn{3}{|l|}{ AJCC stage, 7 th ed* } \\
\hline 0 & $12(6.9 \%)$ & $3(7.9 \%)$ \\
\hline 1 & $12(6.9 \%)$ & $2(5.3 \%)$ \\
\hline ALT, U/L (median, range) & $28(5-1110)$ & $16(3-191)$ \\
\hline CA199, U/mL (median, range) & 36.3 (0.6-6375.0, 1 missing) & $32.4(0.6-474.0)$ \\
\hline CA125, U/mL (median, range) & 22.8 (1.6-742.5, 1 missing) & $16.6(0.6-134.0)$ \\
\hline CEA, ng/mL (median, range) & 3.2 (0.3-285.2, 2 missing) & $6.9(1.9-18.0)$ \\
\hline Fibrinogen, g/L (median, range) & $3.96(1.26-8.56)$ & $3.56(2.01-7.20)$ \\
\hline INR (median, range) & $1.01(0.78-1.92)$ & $0.97(0.68-1.40)$ \\
\hline R0 resection & $91(52.3 \%)$ & $15(39.5 \%)$ \\
\hline 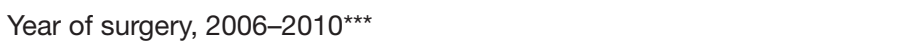 & $85(38.5 \%)$ & $15(39.5 \%)$ \\
\hline No. of events (deaths) & $111(63.8 \%)$ & $25(65.8 \%)$ \\
\hline
\end{tabular}

*, T, N, and AJCC stage were measured with reference to the AJCC staging manual, 7th ed; ${ }^{* *}$, patients with stage 4B were inoperable, so were not involved; ${ }^{\star \star \star}$, dichotomization here is only for data presentation. When indicated in the model, year of surgery was regarded as a continuous variable. 

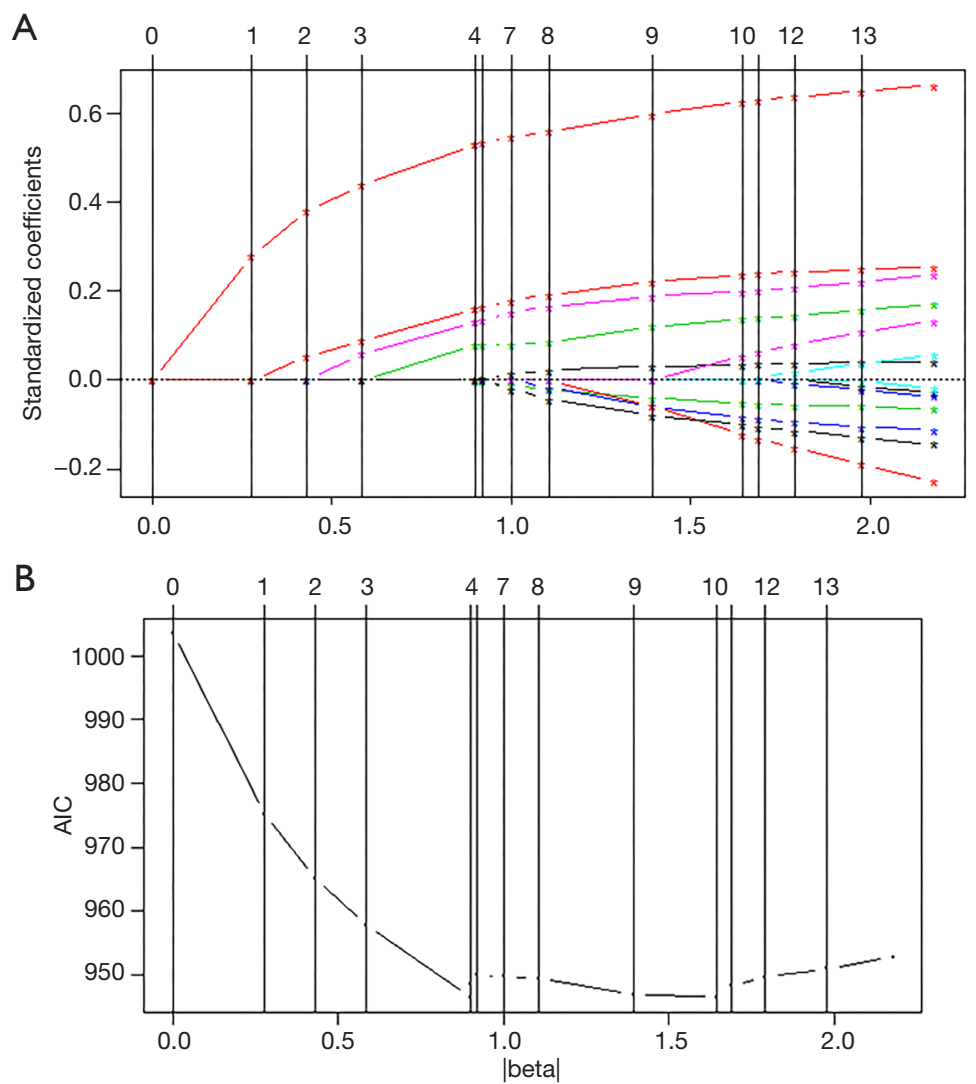

Figure 2 Variable selection using the Lasso regression model. (A) Standardized coefficients in the Lasso procedure. (B) AIC value in the Lasso procedure. The AIC reached the lowest level at 4 and 10 variables. Considering the limitation of the sample size, the step of 4 variables was selected. AIC, Akaike information criterion.

Table 2 Final model derived from the primary cohort

\begin{tabular}{llll}
\hline Predictor & P value & HR & $95 \% \mathrm{Cl}$ \\
\hline T stage, AJCC 7th ed & & & \\
Tis-T1 & & & \\
T2-T4 & 0.0130 & 2.49 & $1.73-3.60$ \\
Lymph node & & & \\
NA & & & \\
Positive & $<0.001$ & 3.97 & $3.19-4.94$ \\
Fibrinogen & 0.0049 & 1.32 & $1.19-1.45$ \\
\hline
\end{tabular}

$\mathrm{Cl}$, confidence interval; HR, hazard ratio.

\section{Correlation between preoperative fibrinogen and clinical variables}

The primary and validation cohorts were dichotomized by the selected cut-off value. As shown in Table 3, older age $(\mathrm{P}=0.01)$, positive lymph nodes $(\mathrm{P}=0.04)$, and two tumor markers $(\mathrm{P}=0.001 \& 0.007)$ significantly correlated with the preoperative fibrinogen level. However, the data in the validation cohort showed a different pattern: only poor differentiation correlated with hyperfibrinogenemia (Table S6). This discrepancy may be explained by the limited size of the validation cohort. Therefore, more in-depth research is needed to fully elucidate this phenomenon.

\section{Survival analysis}

KM curves were drawn to analyze the difference in prognosis between the two groups. Survival analysis of the two groups showed significantly worse outcome in patients with hyperfibrinogenemia, both in the primary cohort and validation cohort (Figure S3). This result strongly indicated a relation between preoperative fibrinogen level and patient prognosis. Based on these results, it seems reasonable to 
Table 3 Demographic characteristics of GBC patients with and without hyperfibrinogenemia

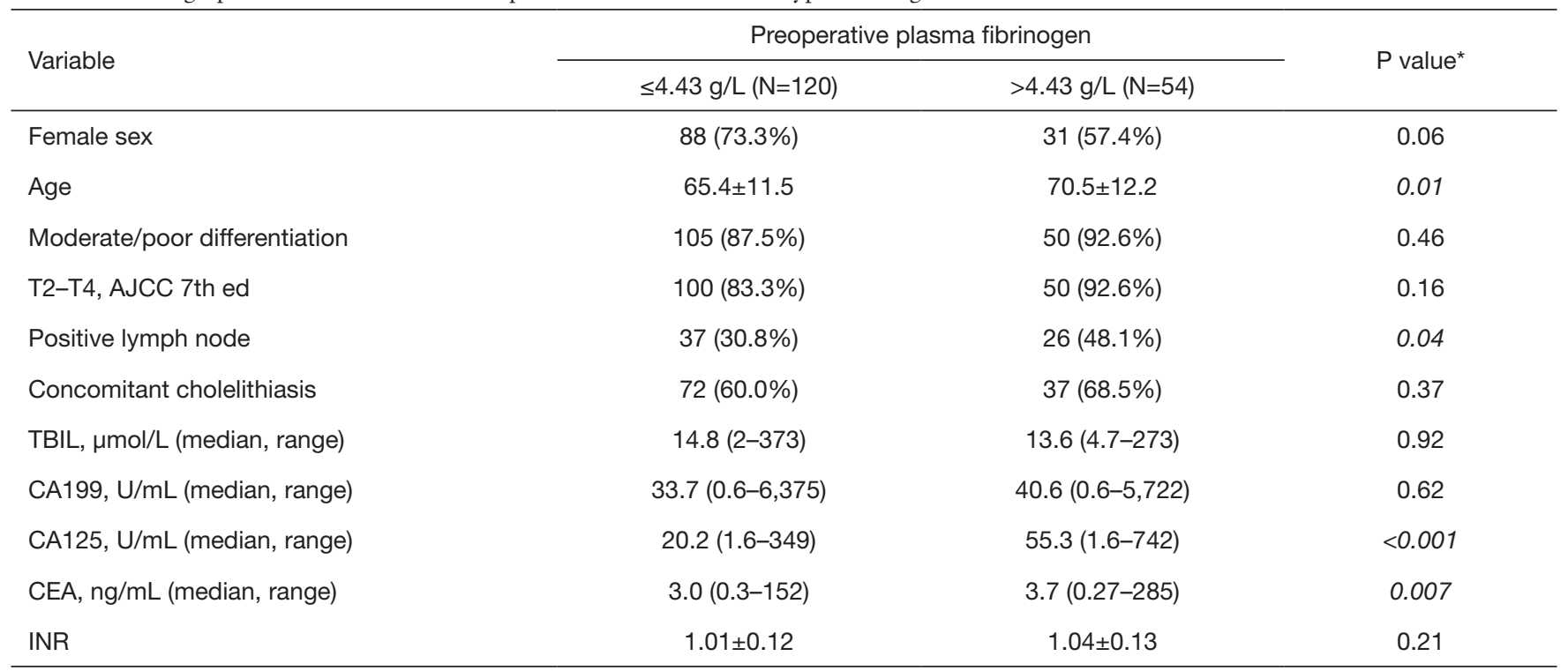

${ }^{*}, \chi^{2}$ test for discrete variables. $T$-test or Mann-Whitney $U$ test for continuous variables. Italic $P$ values indicate $P<0.05$. GBC, gallbladder cancer.

select preoperative fibrinogen as a valid and independent prognostic factor.

\section{Predictive accuracy versus alternative models}

To further validate the value of preoperative fibrinogen, we built alternative prediction models with $\mathrm{T}+\mathrm{N}+\mathrm{X}$, where $\mathrm{T}$ indicated $\mathrm{T}$ stage (7th AJCC), $\mathrm{N}$ indicated lymph node positivity, and $\mathrm{X}$ indicated a third factor other than preoperative fibrinogen. Levels of differentiation, CEA, and CA199 were manually selected in the different alternative models. As shown in Figure 3A, the c-index was significantly higher with our final model compared with the $\mathrm{T}+\mathrm{N}, \mathrm{T}$ $+\mathrm{N}+$ Differentiation, $\mathrm{T}+\mathrm{N}+\mathrm{CEA}$, and $\mathrm{T}+\mathrm{N}+\mathrm{CA} 199$ models. Moreover, there was no significant difference between each of any two alternative models. This result indicated that these alternative factors could not improve the performance of the prediction model.

\section{Model performance}

The performance of the final model was indicated by the c-index. The c-index is the proportion of all pairs of subjects whose survival time can be ordered such that the subject with the higher predicted survival is the one who survives longest. For the primary cohort, the c-index of our nomogram for OS prediction was 0.74 . By the bootstrap validation (with 300 resamples), the c-index of the nomogram was still 0.74 , with optimism $<0.005$, indicating low overfitting. Discrimination of the model was further illustrated by dividing the predictions into four quartiles, and the KM curves were plotted (Figure 3B). Patients in the lower quartile had a considerably poorer prognosis. We then validated our model in the validation cohort. The c-index of our nomogram was 0.71 , indicating general validity of the model and good performance with extrapolation.

\section{Predictive accuracy versus 7th AFCC and Nevin systems}

$\mathrm{KM}$ curves were applied for the survival analysis among different staging systems (Figure 3C,3D). Both the 7th AJCC and Nevin systems showed good prognostic stratification of the patients. However, in our cohort, patients in stage 4 in the 7th AJCC system showed no worse prognosis than those in stage 3. Moreover, the 7th AJCC system was unsatisfactory in stratifying patients between stages 0 and 1 for short-term survival.

The KM curve for our model was based on the four quartiles of patients' risk, as mentioned above. The curve indicated good prognostic stratification, with better accuracy than the two traditional staging systems. The c-index of the nomogram was 0.74 for the primary cohort, and 0.71 for the validation cohort, which is significantly higher $(\mathrm{P}=0.04)$ than the 7 th AJCC system (0.67) and the 


\begin{tabular}{|c|c|c|c|c|c|c|c|}
\hline Predictor & FM & M1 & M2 & M3 & M4 & AJCC $7^{\text {th }}$ stage & Nevin \\
\hline $\begin{array}{l}\text { T stage } \\
\text { (Tis-T1/ T2-T4) }\end{array}$ & $\sqrt{ }$ & $\sqrt{ }$ & $\sqrt{ }$ & $\sqrt{ }$ & $\sqrt{ }$ & - & - \\
\hline $\begin{array}{l}\text { Lymph node } \\
\text { (NA/positive) }\end{array}$ & $\sqrt{ }$ & $\sqrt{ }$ & $\sqrt{ }$ & $\sqrt{ }$ & $\sqrt{ }$ & - & - \\
\hline Fibrinogen & $\sqrt{ }$ & & & & & - & - \\
\hline $\begin{array}{l}\text { Differentiation } \\
\text { (well/mederate/poor) }\end{array}$ & & & $\sqrt{ }$ & & & - & - \\
\hline CA199 & & & & $\sqrt{ }$ & & - & - \\
\hline CEA (3-knot rcs*) & & & & & $\sqrt{ }$ & - & - \\
\hline Degree of freedom & 3 & 2 & 4 & 3 & 4 & 1 & 1 \\
\hline C index (primary cohort) & 0.74 & 0.65 & 0.65 & 0.65 & 0.66 & 0.67 & 0.67 \\
\hline $\mathrm{C}$ index (validation cohort) & 0.71 & 0.67 & 0.67 & 0.56 & 0.67 & & \\
\hline
\end{tabular}

* rcs: restricted cubic spline

B

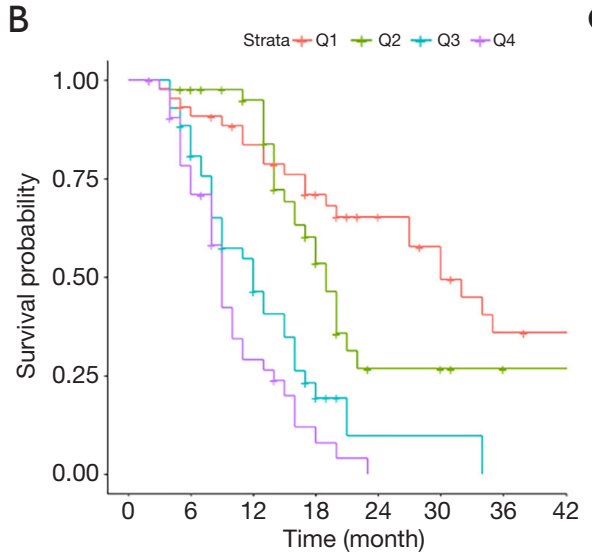

C

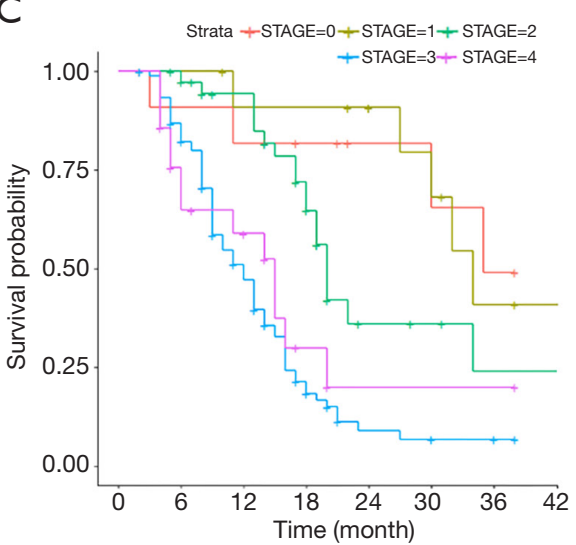

D

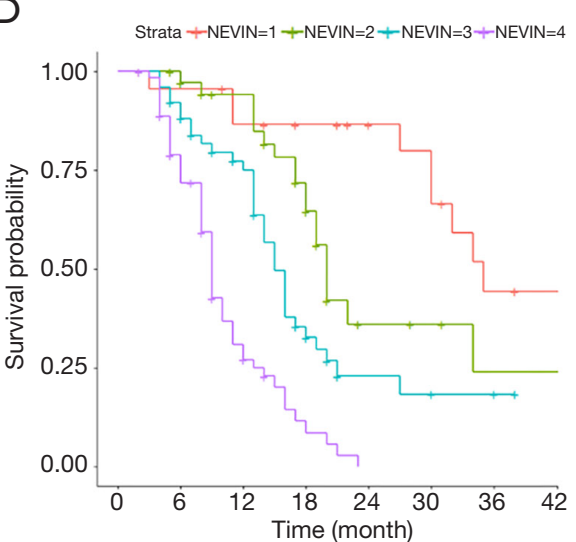

Figure 3 Model comparison. (A) Different c-index of different models. As model development was not needed for AJCC stage and Nevin stage, the two cohorts were measured together. (B-D) KM curves of (B) the quartiles of our final model; (C) stages in AJCC 7th system; (D) stages in the Nevin system.

Nevin system (0.67). No significant difference was found between the 7th AJCC and Nevin systems. The results suggested that our model was a practical and valuable tool for predicting OS.

\section{Model presentation}

The results of the modeling were presented as a nomogram. The nomogram shown in Figure $4 A$ is a practical tool for clinical use to calculate the prognosis of patients. Figure $4 B$ shows the relative contribution of each predictor. The calibration curves are shown in Figure $4 C$ and $4 D$.

\section{Discussion}

Clinical prediction models are becoming increasingly popular because of (I) their ability to incorporate continuous variables into prognosis; (II) their ability to estimate individualized risk; and (III) their userfriendly interface (33). A number of clinical prediction tools are being used for bladder, breast, and pancreatic cancers, intrahepatic cholangiocarcinoma, perihilar cholangiocarcinoma, and other malignancies. But a tool for the prediction of prognosis of GBC patient has been absent, likely because of the low incidence of GBC. Here we have presented the first factor analysis of GBC patients who underwent surgical resection, and developed and validated a nomogram for prognosis prediction.

We analyzed potential predictors mentioned in the literature. Based on objective criteria, T stage, lymph node metastasis, and preoperative fibrinogen level were included in the model. $\mathrm{T}$ stage was clustered into two 
A
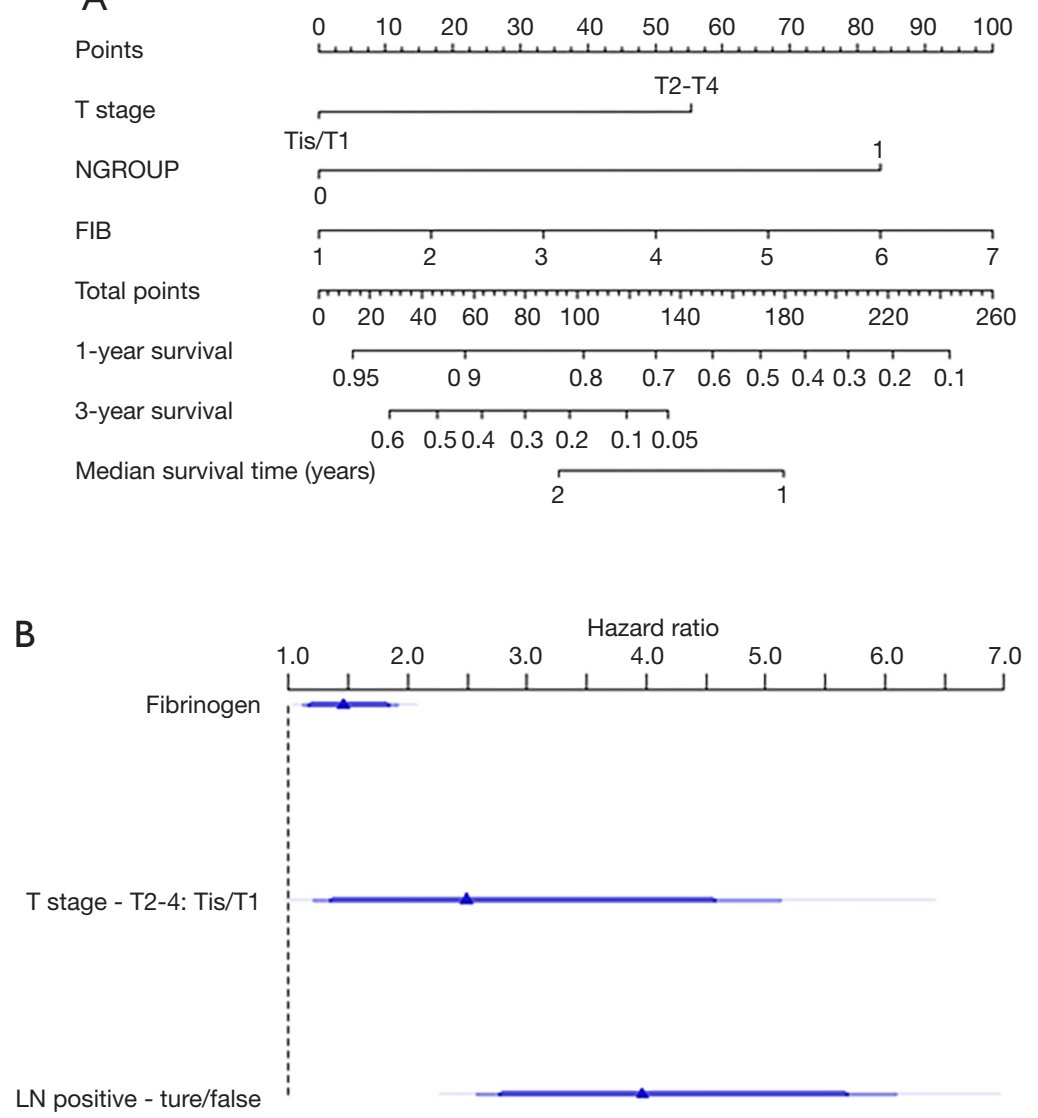

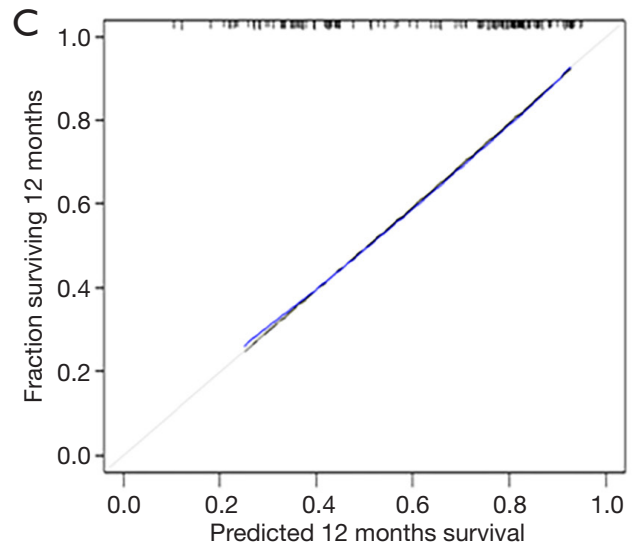

D

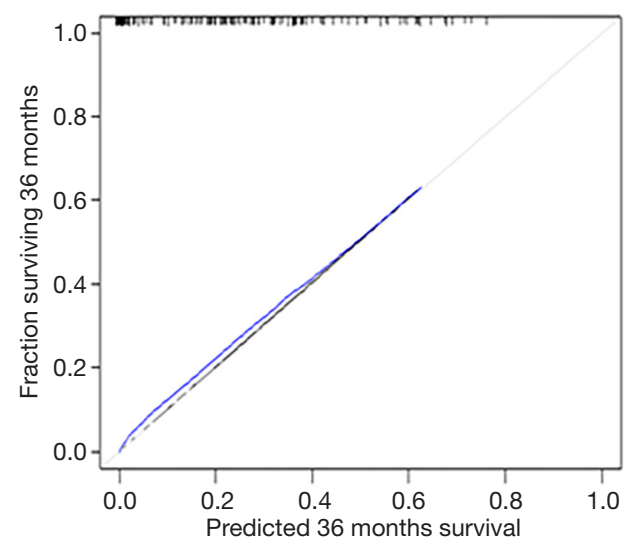

Figure 4 Prediction nomogram. (A) The prediction model presented as a nomogram; (B) contribution of each of the three predictors shown with the hazard ratio; (C,D) Bootstrap estimate of calibration accuracy for 1-year (C) and 3-year (D) estimates from the final Cox model. The blue curve corresponds to bootstrap-corrected estimates.

groups: tumor invades no deeper than the muscular layer or not (namely, T0-T1 or T2-T4a, 7th AJCC). At the same time, lymph node metastasis was shown to be a strong indicator of prognosis. In addition, preoperative fibrinogen was indicated to be a better-performing predictor than the others: level of differentiation, concomitant cholelithiasis, preoperative jaundice (indicated by total bilirubin), and classical tumor markers (CA-199, CA-125, and CEA).

As an essential part of the hemostatic system, fibrinogen levels have been related to the prognosis of various types of solid tumors (10). Our team previously found that preoperative hyperfibrinogenemia was an independent predictor of poor prognosis in GBC patients (13). In this current study, we proposed that the preoperative serum fibrinogen level, together with $\mathrm{T}$ and $\mathrm{N}$ staging, is a valuable predictor of the prognosis of GBC patients.

As shown above, based on a backward stepwise selection and a Lasso procedure respectively, we obtained comparable results for the included predictors. To examine the relevance of each predictor with clinical significance, three selected variables were analyzed separately. After both statistical and clinical significance was verified, the model's performance was analyzed with the validation cohort, then in comparison with two classical staging systems. The c-index indicated that our model had better performance. Moreover, the redundancy analysis showed that the fibrinogen level had little dependence with either $\mathrm{T}$ or $\mathrm{N}$ stage. It is thus reasonable to suggest that fibrinogen is an independent predictor, although the mechanism is not well understood.

We also tried to develop a prediction model for lymph 
node metastasis using a similar procedure, but no variable was detected to have a significant predictive effect.

The underlying mechanism of the relationship between plasma hyperfibrinogenemia and poor prognosis has not been fully elucidated. Proliferation, EMT, migration, angiogenesis, and hematogenous metastasis are observed to be correlated with fibrinogen $(9,13,34)$. Our team previously found that GBC cell lines cocultured with fibrinogen showed an increased degree of EMT (13). Moreover, fibrinogen is a key regulator of inflammation in various diseases. Cross-talk exists between coagulation, inflammation, and cancer development $(35,36)$. More indepth research is needed to understand the molecular mechanisms in this process.

\section{Study limitations}

Because GBC is a rare disease, the sample size was limited to a single center. Though our study showed the significance of preoperative fibrinogen in the prediction of prognosis, a larger volume, multicenter study is needed to validate our results. In the cohort of more than 10 years, radiotherapy was not performed in our hospital, but systematic chemotherapy was applied in the last few years. Thus, chemotherapy could not be included in the model because there were too many missing values. However, this effect was indirectly testified by the predictor 'operation year', which showed no significant effect. In some cases, our model was still not very effective in predicting the prognosis and the underlying reason needs more research to unveil it. The study was internally limited by its retrospective design, so external validation and the bootstrap procedure were applied to improve the validity of the results.

In conclusion, this study presents a practical nomogram to predict the prognosis of GBC patients after operation. Fibrinogen is a valuable predictor of the OS of GBC patients.

\section{Acknowledgments}

Funding: This study was supported by the National Natural Science Foundation of China (81974371), the Shanghai "Rising Stars of Medical Talent" Youth Development Program (Youth Medical Talents 2019 No. 72), the National Science and Technology Major Project (2019ZX09301-158), and the Shanghai Anticancer Association Eyas Program (SACA-CY19C17).

\section{Footnote}

Reporting Checklist: The authors have completed the TRIPOD reporting checklist. Available at https://dx.doi. org/10.21037/jgo-21-357

Data Sharing Statement: Available at https://dx.doi. org/10.21037/jgo-21-357

Conflicts of Interest: All authors have completed the ICMJE uniform disclosure form (available at https://dx.doi. org/10.21037/jgo-21-357). The authors have no conflicts of interest to declare.

Ethical Statement: The authors are accountable for all aspects of the work in ensuring that questions related to the accuracy or integrity of any part of the work are appropriately investigated and resolved. All procedures performed in this study involving human participants were in accordance with the Declaration of Helsinki (as revised in 2013). It was approved by the Committee for Ethics of Xinhua Hospital Affiliated to Shanghai Jiao Tong University School of Medicine (No. XHEC-C-2018-174). Informed consent was given before surgery.

Open Access Statement: This is an Open Access article distributed in accordance with the Creative Commons Attribution-NonCommercial-NoDerivs 4.0 International License (CC BY-NC-ND 4.0), which permits the noncommercial replication and distribution of the article with the strict proviso that no changes or edits are made and the original work is properly cited (including links to both the formal publication through the relevant DOI and the license). See: https://creativecommons.org/licenses/by-nc-nd/4.0/.

\section{References}

1. Li M, Zhang Z, Li X, et al. Whole-exome and targeted gene sequencing of gallbladder carcinoma identifies recurrent mutations in the ErbB pathway. Nat Genet 2014;46:872-6.

2. Surveillance, Epidemiology, and End Results (SEER) Program (www.seer.cancer.gov) Research Data (19732014), National Cancer Institute, DCCPS, Surveillance Research Program, released April 2017, based on the November 2016 submission.

3. Hundal R, Shaffer EA. Gallbladder cancer: epidemiology and outcome. Clin Epidemiol 2014;6:99-109. 
4. Randi G, Franceschi S, La Vecchia C. Gallbladder cancer worldwide: geographical distribution and risk factors. Int J Cancer 2006;118:1591-602.

5. Misra S, Chaturvedi A, Misra NC, et al. Carcinoma of the gallbladder. Lancet Oncol 2003;4:167-76.

6. Zhang F, Ma Q, Xu Z, et al. Dihydroartemisinin inhibits TCTP-dependent metastasis in gallbladder cancer. J Exp Clin Cancer Res 2017;36:68.

7. Boutros C, Gary M, Baldwin K, et al. Gallbladder cancer: past, present and an uncertain future. Surg Oncol 2012;21:e183-91.

8. Boccaccio C, Sabatino G, Medico E, et al. The MET oncogene drives a genetic programme linking cancer to haemostasis. Nature 2005;434:396-400.

9. Guglietta S, Chiavelli A, Zagato E, et al. Coagulation induced by $\mathrm{C} 3 \mathrm{aR}$-dependent NETosis drives protumorigenic neutrophils during small intestinal tumorigenesis. Nat Commun 2016;7:11037.

10. Perisanidis C, Psyrri A, Cohen EE, et al. Prognostic role of pretreatment plasma fibrinogen in patients with solid tumors: A systematic review and meta-analysis. Cancer Treat Rev 2015;41:960-70.

11. Seebacher V, Polterauer S, Grimm C, et al. The prognostic value of plasma fibrinogen levels in patients with endometrial cancer: a multi-centre trial. Br J Cancer 2010;102:952-6.

12. Guo Q, Zhang B, Dong X, et al. Elevated levels of plasma fibrinogen in patients with pancreatic cancer: possible role of a distant metastasis predictor. Pancreas 2009;38:e75-9.

13. Shu YJ, Weng H, Bao RF, et al. Clinical and prognostic significance of preoperative plasma hyperfibrinogenemia in gallbladder cancer patients following surgical resection: a retrospective and in vitro study. BMC Cancer 2014;14:566.

14. Oh TG, Chung MJ, Bang S, et al. Comparison of the sixth and seventh editions of the AJCC TNM classification for gallbladder cancer. J Gastrointest Surg 2013;17:925-30.

15. Kayahara M, Nagakawa T, Nakagawara H, et al. Prognostic factors for gallbladder cancer in Japan. Ann Surg 2008;248:807-14.

16. Liska V, Treska V, Skalicky T, et al. Evaluation of Tumor Markers and Their Impact on Prognosis in Gallbladder, Bile Duct and Cholangiocellular Carcinomas - A Pilot Study. Anticancer Res 2017;37:2003-9.

17. Muszynska C, Lundgren L, Lindell G, et al. Predictors of incidental gallbladder cancer in patients undergoing cholecystectomy for benign gallbladder disease: Results from a population-based gallstone surgery registry. Surgery 2017;162:256-63.
18. Higuchi R, Ota T, Araida T, et al. Surgical approaches to advanced gallbladder cancer : a 40-year single-institution study of prognostic factors and resectability. Ann Surg Oncol 2014;21:4308-16.

19. Iasonos A, Schrag D, Raj GV, et al. How to build and interpret a nomogram for cancer prognosis. J Clin Oncol 2008;26:1364-70.

20. Wang Y, Li J, Xia Y, et al. Prognostic nomogram for intrahepatic cholangiocarcinoma after partial hepatectomy. J Clin Oncol 2013;31:1188-95.

21. Groot Koerkamp B, Wiggers JK, Gonen M, et al. Survival after resection of perihilar cholangiocarcinomadevelopment and external validation of a prognostic nomogram. Ann Oncol 2016;27:753.

22. Huang YQ, Liang CH, He L, et al. Development and Validation of a Radiomics Nomogram for Preoperative Prediction of Lymph Node Metastasis in Colorectal Cancer. J Clin Oncol 2016;34:2157-64.

23. Tendulkar RD, Agrawal S, Gao T, et al. Contemporary Update of a Multi-Institutional Predictive Nomogram for Salvage Radiotherapy After Radical Prostatectomy. J Clin Oncol 2016;34:3648-54.

24. Wu W, Dong P, Wu X, et al. Three-step method for systematic lymphadenectomy in gastric cancer surgery using the 'curettage and aspiration dissection technique' with Peng's multifunctional operative dissector. World J Surg Oncol 2014;12:322.

25. Edge SB. American Joint Committee on Cancer, American Cancer Society. AJCC cancer staging handbook: from the AJCC cancer staging manual. 7th ed. New York: Springer, 2010.

26. Harrell FEJr. Regression Modeling Strategies. 2 ed. Switzerland: Springer International Publishing, 2015.

27. Harrell FE Jr. rms: Regression Modeling Strategies. R package version 5.1-2. 2018.

28. Steyerberg EW. Clinical Prediction Models. Springer, 2009.

29. Kassambara A, Kosinski M. survminer: Drawing Survival Curves using 'ggplot2'. R package version 0.4.2. 2018.

30. Park MY, Hastie T. glmpath: L1 Regularization Path for Generalized Linear Models and Cox Proportional Hazards Model. R package version 0.98. 2018.

31. Wickham H. tidyverse: Easily Install and Load the 'Tidyverse'. R package version 1.2.1. 2017.

32. Ito H, Ito K, D'Angelica $M$, et al. Accurate staging for gallbladder cancer: implications for surgical therapy and pathological assessment. Ann Surg 2011;254:320-5.

33. Balachandran VP, Gonen M, Smith JJ, et al. Nomograms 
in oncology: more than meets the eye. Lancet Oncol 2015;16:e173-80.

34. Steinbrecher KA, Horowitz NA, Blevins EA, et al. Colitisassociated cancer is dependent on the interplay between the hemostatic and inflammatory systems and supported by integrin alpha(M)beta(2) engagement of fibrinogen. Cancer Res 2010;70:2634-43.

Cite this article as: Yang Z, Ren T, Liu S, Cai C, Gong W, Shu Y. Preoperative serum fibrinogen as a valuable predictor in the nomogram predicting overall survival of postoperative patients with gallbladder cancer. J Gastrointest Oncol 2021;12(4):1661-1672. doi: 10.21037/jgo-21-357
35. Grivennikov SI, Greten FR, Karin M. Immunity, inflammation, and cancer. Cell 2010;140:883-99.

36. Davalos D, Akassoglou K. Fibrinogen as a key regulator of inflammation in disease. Semin Immunopathol 2012;34:43-62.

(English Language Editor: K. Brown) 
Supplementary

Table S1 Steyerberg checklist

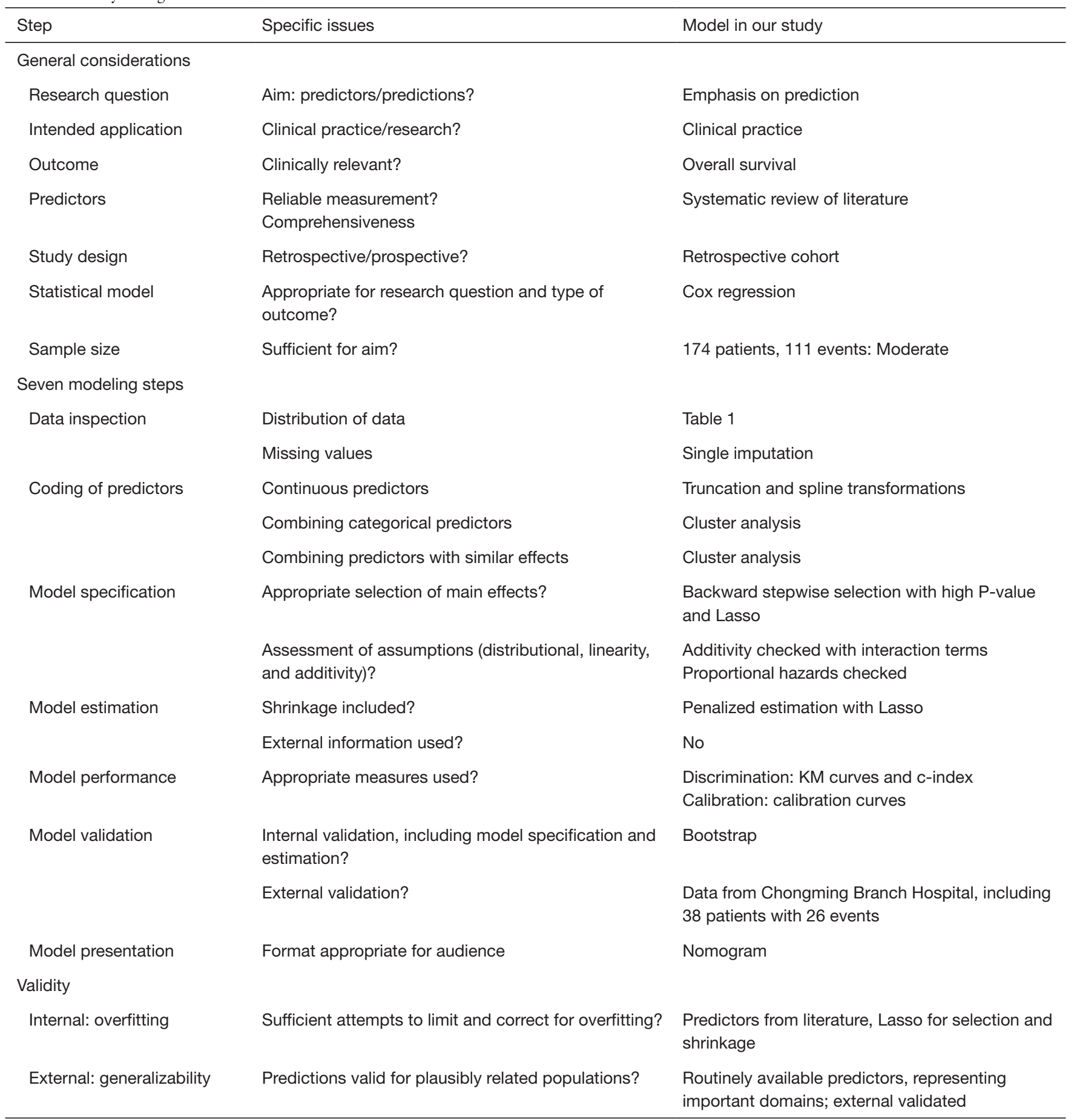

(C) Journal of Gastrointestinal Oncology. All rights reserved. 
Table S2 Cox regression coefficients in the full model

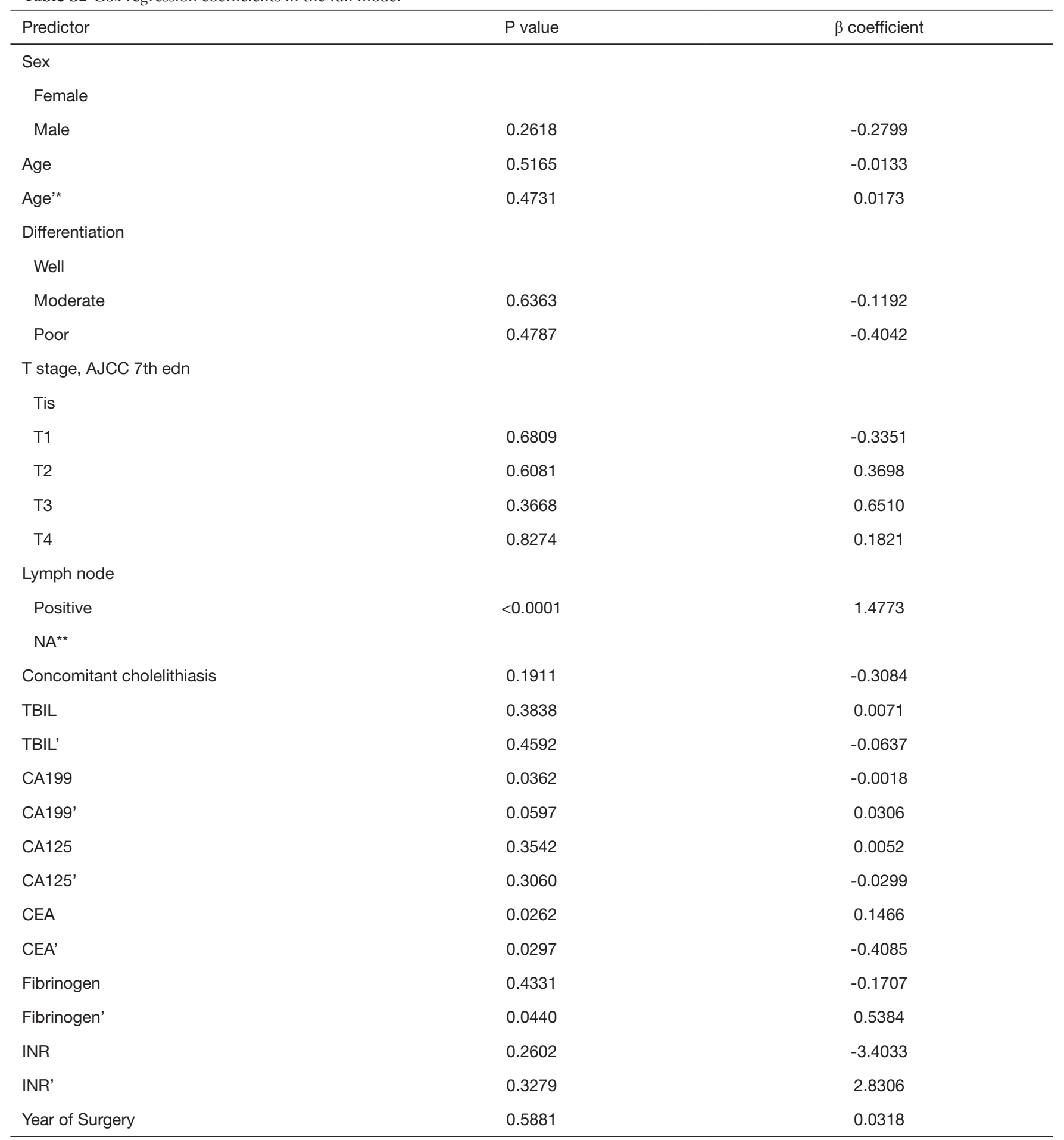

*Continuous variables were fitted with restricted cubic spline functions, requiring 2 independent coefficients respectively, like: AGE, AGE'. ${ }^{* \star} A$ s the " $N$ negative and nodes examined $\geq 6$ " group was too small to analyze, it was merged with the " $N$ negative and nodes examined $<6$ " group, and renamed "not available". 


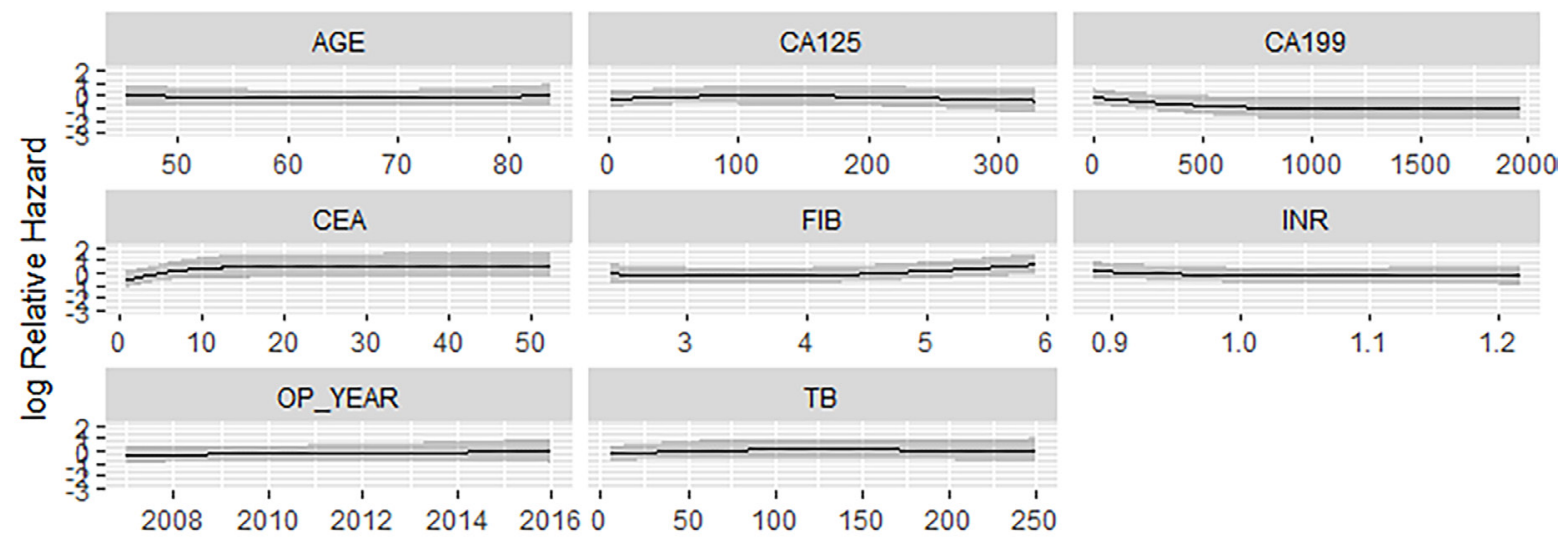

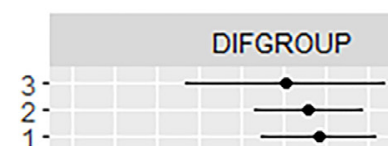

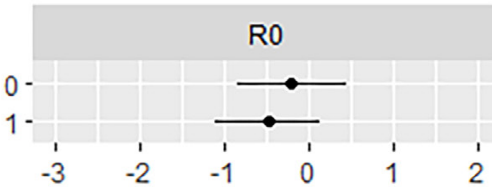

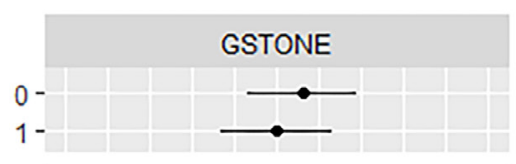

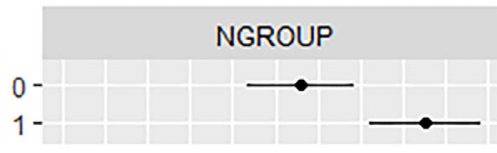

M-

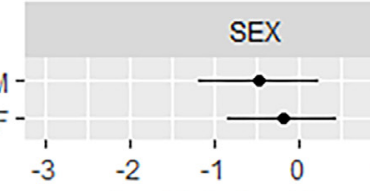

log Relative Hazard

Figure S1 Trend of predictors on log hazard of death in the full model. DIFGROUP: level of differentiation, 1-well, 2-moderate, 3-poor. GSTONE: concomitant cholestasis, 1-positive, 0-negative. NGROUP: lymph node involved, 1-positive, 0-not available. R0: 1-margin negative, 0 -margin positive. SEX: M-male, F-female. 
Table S3 Cox regression coefficients in the simplified full model, then the stepwise selection and Lasso results

\begin{tabular}{|c|c|c|c|c|c|}
\hline Predictor & \multicolumn{2}{|c|}{ Simplified full model } & \multicolumn{2}{|c|}{ Stepwise (AIC) } & $\begin{array}{c}\text { Lasso } \\
\beta \text { coefficient }\end{array}$ \\
\hline Sex & & & Not selected & & Not selected \\
\hline \multicolumn{6}{|l|}{ Female } \\
\hline Male & 0.7136 & -0.0789 & & & \\
\hline Differentiation & & & Not selected & & Not selected \\
\hline \multicolumn{6}{|l|}{ Well } \\
\hline Moderate/poor & 0.6472 & -0.1968 & & & \\
\hline \multicolumn{6}{|l|}{ T stage, AJCC 7th edn } \\
\hline \multicolumn{6}{|l|}{ Lymph node } \\
\hline Positive & $<0.0001$ & 1.4155 & $<0.001$ & 1.3839 & 1.10 \\
\hline \multicolumn{6}{|l|}{$N A^{*}$} \\
\hline Concomitant cholelithiasis & 0.3015 & -0.2250 & Not selected & & Not selected \\
\hline TBIL & 0.2346 & 0.0018 & Not selected & & Not selected \\
\hline CA199 & 0.0941 & 0.0002 & Not selected & & Not selected \\
\hline CA125 & 0.2610 & 0.0014 & Not selected & & 0.0006 \\
\hline CEA & 0.7561 & 0.0008 & Not selected & & Not selected \\
\hline \multicolumn{6}{|c|}{$\begin{array}{l}\text { *As the "N negative and nodes examined } \geq 6 \text { " group was too small to analyze, it was merged with the "N negative and nodes examine } \\
<6 \text { " group, and renamed "not available". }\end{array}$} \\
\hline \multicolumn{2}{|l|}{ Predictor } & \multicolumn{2}{|l|}{$\chi^{2}$} & \multicolumn{2}{|l|}{$P$ value } \\
\hline \multicolumn{2}{|l|}{ T stage (T0-1/T2-4) } & \multicolumn{2}{|l|}{0.8363} & \multicolumn{2}{|l|}{0.360} \\
\hline \multicolumn{2}{|l|}{ Positive lymph node } & \multicolumn{2}{|l|}{0.0092} & \multicolumn{2}{|l|}{0.924} \\
\hline \multicolumn{2}{|l|}{ Fibrinogen } & \multicolumn{2}{|l|}{0.0084} & \multicolumn{2}{|l|}{0.927} \\
\hline \multicolumn{2}{|l|}{ Global } & \multicolumn{2}{|l|}{0.879} & \multicolumn{2}{|l|}{0.831} \\
\hline
\end{tabular}


Table S5 Redundancy analysis of final model

\begin{tabular}{lc}
\hline Predictor & $\mathrm{R}^{2}$ \\
\hline T stage & 0.158 \\
Positive lymph node & 0.144 \\
Fibrinogen & 0.071 \\
\hline
\end{tabular}

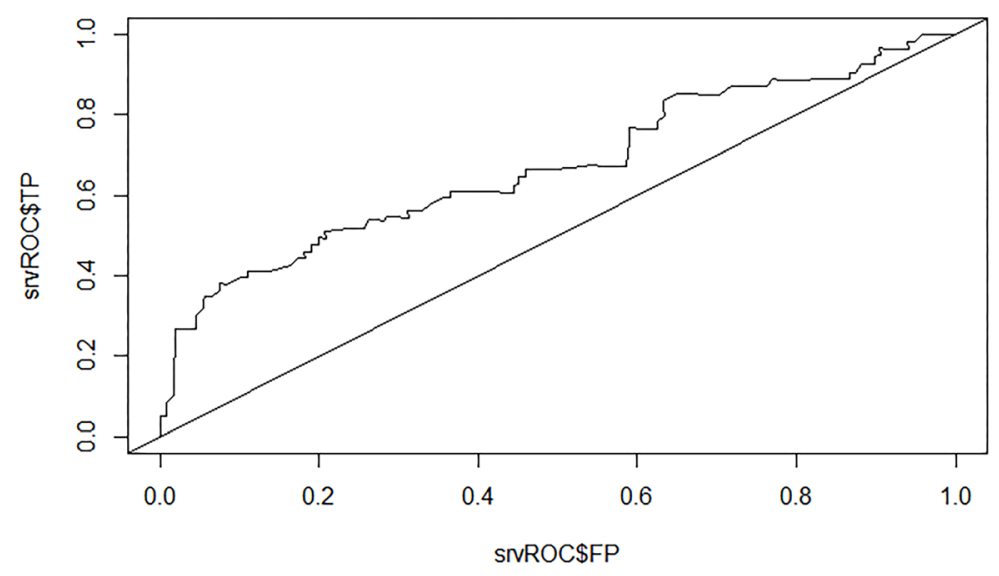

Figure S2 ROC of fibrinogen on predicting the prognosis (year =3).

Table S6 Demographic characteristics of GBC patients of validation cohort with and without hyperfibrinogenemia

\begin{tabular}{|c|c|c|c|}
\hline Variable & \multicolumn{2}{|c|}{ Preoperative plasma fibrinogen } & P-value* \\
\hline Female sex & 20 & 9 & 0.93 \\
\hline Age & $68.3 \pm 12.7$ & $62.0 \pm 11.2$ & 0.14 \\
\hline Moderate/Poor Differentiation & 23 & 10 & $<0.01$ \\
\hline Positive lymph node & 8 & 4 & 0.98 \\
\hline Concomitant cholelithiasis & 21 & 7 & 0.62 \\
\hline TBIL, $\mu \mathrm{mol} / \mathrm{L}$ (median, range) & $14.0(7-244)$ & $12.0(7-43)$ & 0.50 \\
\hline CA199, U/mL (median, range) & $31.0(0.6-474)$ & $37.6(0.6-51.6)$ & 0.69 \\
\hline INR & $0.97 \pm 0.15$ & $0.97 \pm 0.12$ & 0.94 \\
\hline
\end{tabular}

${ }^{*} \chi^{2}$ test for discrete variables. $T$-test or Mann-Whitney $U$ test for continuous variables. 

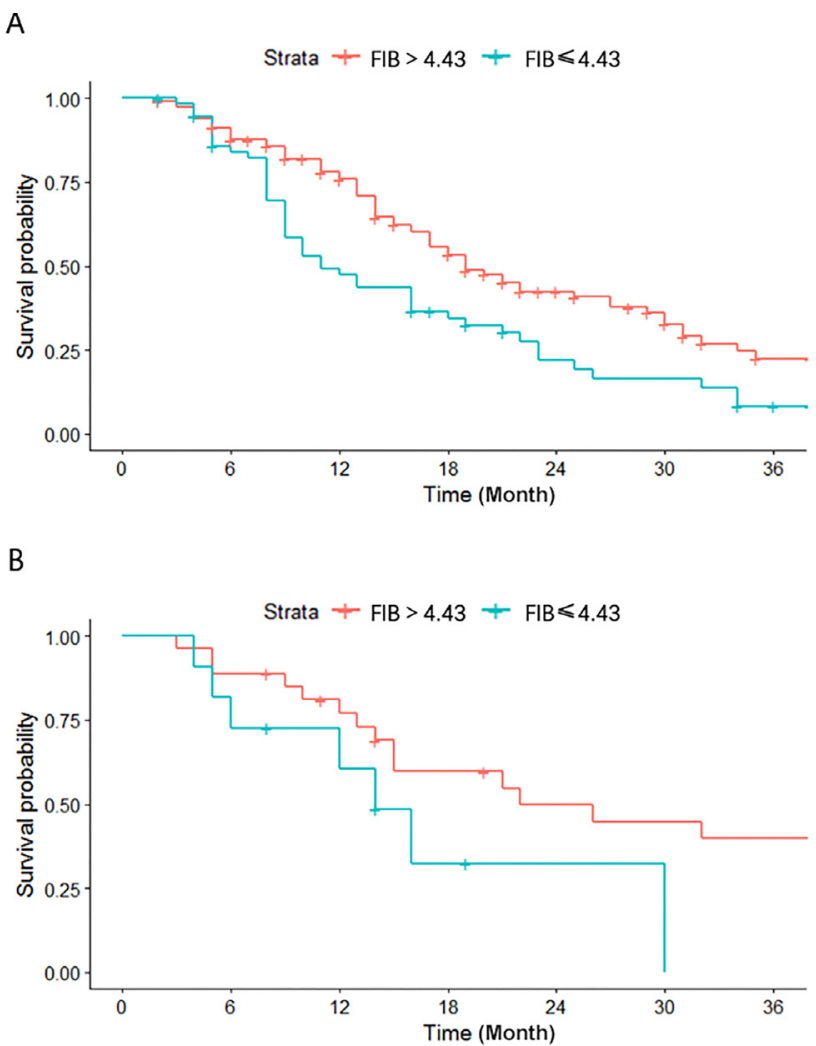

Figure S3 Survival analysis of the group dichotomized by preoperative serum fibrinogen level. (A) KM curve of the primary cohort. (B) KM curve of the validation cohort. 